\title{
TRIANGLE-ROUNDEDNESS IN MATROIDS
}

\author{
JOÃO PAULO COSTALONGA ${ }^{1}$ AND XIANGQIAN ZHOU ${ }^{2}$
}

\begin{abstract}
A matroid $N$ is said to be triangle-rounded in a class of matroids $\mathscr{M}$ if each 3connected matroid $M \in \mathscr{M}$ with a triangle $T$ and an $N$-minor has an $N$-minor with $T$ as triangle. Reid gave a result useful to identify such matroids as stated next: suppose that $M$ is a binary 3-connected matroid with a 3-connected minor $N, T$ is a triangle of $M$ and $e \in T \cap E(N)$; then $M$ has a 3-connected minor $M^{\prime}$ with an $N$-minor such that $T$ is a triangle of $M^{\prime}$ and $\left|E\left(M^{\prime}\right)\right| \leq|E(N)|+2$. We strengthen this result by dropping the condition that such element $e$ exists

and proving that there is a 3-connected minor $M^{\prime}$ of $M$ with an $N$-minor $N^{\prime}$ such that $T$ is a triangle of $M^{\prime}$ and $E\left(M^{\prime}\right)-E\left(N^{\prime}\right) \subseteq T$. This result is extended to the non-binary case and, as an application, we prove that $M\left(K_{5}\right)$ is triangle-rounded in the class of the regular matroids.
\end{abstract}

Key words: matroid minors; roundedness; matroid connectivity.

\section{INTRODUCTION}

Let $\mathscr{M}$ be a class of matroids closed for minors and isomorphisms and let $\mathscr{F}$ be a family of matroids. An $\mathscr{F}$-minor of a matroid $M$ is a minor of $M$ isomorphic to a member of $\mathscr{F}$. A matroid $M$ uses a set $T$ if $T \subseteq E(M)$. We say that $\mathscr{F}$ is $(k, t)$-rounded in $\mathscr{M}$ if each element of $\mathscr{F}$ is $k$-connected and, for each $k$-connected matroid $M \in \mathscr{M}$ with an $\mathscr{F}$-minor and each $t$-subset $T \subseteq E(M), M$ has an $\mathscr{F}$-minor using $T$. We define $\mathscr{F}$ to be $t$-rounded in $\mathscr{M}$ if it is $(t+1, t)$-rounded in $\mathscr{M}$. A matroid $N$ is said $(k, t)$-rounded (resp. $t$-rounded) in $\mathscr{M}$ if so is $\{N\}$. When we simply say that a matroid or family of matroids is $(k, t)$-rounded or $t$-rounded with no mention to a specific class of matroids, we are referring to the class of all matroids.

Bixby [1] proved that $U_{2,4}$ is 1-rounded. Seymour [14] established a method to find a minimal 1-rounded family containing a given family of matroids; in that work it is established that $\left\{U_{2,4}, M\left(K_{4}\right)\right\},\left\{U_{2,4}, F_{7}, F_{7}^{*}\right\},\left\{U_{2,4}, F_{7}, F_{7}^{*}, M^{*}\left(K_{3,3}\right), M^{*}\left(K_{5}\right), M^{*}\left(K_{3,3}^{\prime}\right)\right\}$ and $\left\{U_{2,5}, U_{3,5}, F_{7}, F_{7}^{*}\right\}$ are 1-rounded.

Seymour [16] proved that $U_{2,4}$ is also 2-rounded and, later, in [17], established a method to find a minimal 2-rounded family containing a given family of matroids.

Khan [6] and Coullard [5] proved independently that $U_{2,4}$ is not 3-rounded. To the best of our knowledge, there is no known criterion to check $(k, t)$-roundedness for $k \geq 4$. For $t \geq 3$, Oxley [9] proved that $\left\{U_{2,4}, \mathscr{W}^{3}\right\}$ is $(3,3)$-rounded. Moss [7] proved that $\left\{\mathscr{W}^{2}, \mathscr{W}^{3}, \mathscr{W}^{4}, M\left(\mathscr{W}_{3}\right), M\left(\mathscr{W}_{4}\right), Q_{6}\right\}$

\footnotetext{
${ }^{1}$ Departamento de Matemática, Universidade Federal do Espírito Santo. Av. Fernando Ferrari, 514; Campus de Goiabeiras, 29075-910, Vitória, ES, Brazil. e-mail: joaocostalonga@gmail.com (corresponding author).

${ }^{2}$ Department of Mathematics and Statistics, Wright State University, Dayton, OH, 45435, USA and School of Mathematical Sciences, Huaqiao University, Fujian, China. e-mail: xiangqian.zhou@wright.edu.
} 
is $(3,4)$-rounded and $\left\{M\left(\mathscr{W}_{3}\right), M\left(\mathscr{W}_{4}\right), M\left(\mathscr{W}_{5}\right), M\left(K_{5} \backslash e\right), M^{*}\left(K_{5} \backslash e\right), M\left(K_{1,2,3}\right), M^{*}\left(K_{1,2,3}\right), S_{8}\right\}$ is $(3,5)$ rounded in the class of the binary matroids.

There are results on classification of small $t$-rounded families of matroids for $t=1,2$. Oxley [8] proved that for $|E(N)| \geq 4, N$ is 1-rounded if and only if $N \cong U_{2,4}, P\left(U_{1,3}, U_{1,1}\right)$ or $Q_{6}$ and 2-rounded if and only if $N \cong U_{2,4}$. Reid and Oxley [12] proved that, up to isomorphisms, the unique 2-rounded matroids with more than three members in the class of $G F(q)$-representable matroids are $M\left(\mathscr{W}_{3}\right)$ and $\mathscr{M}\left(\mathscr{W}_{4}\right)$ for $q=2, U_{2,4}$ and $\mathscr{W}^{3}$ for $q=3$ and $U_{2,4}$ for $q \geq 4$.

In this work, we focus on a different type of roundedness. A family of matroids $\mathscr{F}$ is said to be triangle-rounded in $\mathscr{M}$ if all members of $\mathscr{F}$ are 3 -connected and, for each matroid $M \in \mathscr{M}$ with an $\mathscr{F}$-minor and each triangle $T$ of $M$, there is an $\mathscr{F}$-minor of $M$ with $T$ as triangle. We say that a matroid $N$ is triangle-rounded in $\mathscr{M}$ if so is $\{N\}$. Some examples of trianglerounded matroids and families are $U_{2,4}$ in the class of all matroids, $F_{7}$ in the class of binary matroids and $M^{*}\left(K_{3,3}\right)$ in the class of regular matroids (Asano, Nishizeki and Seymour [2]), $M\left(K_{5} \backslash e\right)$ in the class of regular matroids and $\left\{S_{8}, J_{10}\right\}$ in the class of binary matroids (Reid [13]). The proofs for the triangle-roundedness of the later two rely on the following criterion:

Theorem 1. (Reid [13, Theorem 1.1]) Let $\{e, f, g\}$ be a triangle of a 3 -connected binary matroid $M$ and $N$ be a 3-connected minor of $M$ with $e \in E(N)$. Then, there exists a 3-connected minor $M^{\prime}$ of $M$ using $\{e, f, g\}$ such that $M^{\prime}$ has a minor which is isomorphic to $N$ and $E\left(M^{\prime}\right)$ has at most $|E(N)|+2$ elements.

Here we establish a stronger result for binary matroids:

Theorem 2. If $M$ is a 3-connected binary matroid with a 3-connected minor $N$ and $T$ is a triangle of $M$, then $M$ has a 3-connected minor $M^{\prime}$ using $T$ with an $N$-minor $N^{\prime}$ such that $E\left(M^{\prime}\right)-E\left(N^{\prime}\right) \subseteq T$.

Theorem 2 will be proved using a stronger result:

Theorem 3. Let $M$ be a matroid with a 3-connected minor $N$ satisfying $|E(N)| \geq 4$. Suppose that $T$ is a triangle of $M$ and $M$ is minor-minimal with the property that $M$ is 3-connected and has an $N$-minor using $T$. Then $r(M)-r(N) \leq 2$ and for some $N$-minor $N^{\prime}$ of $M, \mid E(M)-$ $\left(E\left(N^{\prime}\right) \cup T\right) \mid \leq 1$. Moreover, if $E(M)-E\left(N^{\prime}\right) \nsubseteq T$, then one of the following assertions holds:

(a) $r(M)-r(N)=1, r^{*}(M)-r^{*}(N) \in\{2,3\}$ and $M$ has an element $x$ such that $E(M)-E\left(N^{\prime}\right) \subseteq$ $T \cup x, T \cup x$ is a 4-point line of $M, M \backslash x$ has no $N$-minor and $x$ is the unique element of $M$ such that $\operatorname{si}(M / x)$ is 3-connected with an $N$-minor; or

(b) $r^{*}(M)-r^{*}(N)=2, r(M)-r(N) \in\{1,2\}$ and $M$ has an element $y$ such that $E(M)-E\left(N^{\prime}\right) \subseteq$ $T \cup y, T \cup y$ is a 4-cocircuit of $M, M / y$ has no $N$-minor and $M \backslash A$ has no $N$-minor for each subset $A$ of $T$ with $|A| \geq 2$.

All the possible cases described in this theorem indeed occur, we give examples in Section 3. We say that a graph $G$ is triangle-rounded if so is $M(G)$ in the class of graphic matroids. Using Theorem 2 , we establish that $K_{5}$ is triangle-rounded, in other words:

Theorem 4. If $G$ is a 3-connected graph with a triangle $T$ and a $K_{5}$-minor, then $G$ has a $K_{5}$ minor with $E(T)$ as edge-set of a triangle. 
Remark: $K_{3}$ and $K_{4}$ are triangle-rounded, but no larger complete graph than $K_{5}$ is trianglerounded. Indeed, consider, for disjoint sets $X, Y$ and $\{z\}$ satisfying $|X|,|Y| \geq 2$, a complete graph $K$ with $n$ vertices with $X \cup Y \cup\{z\}$ as vertex set. Consider also a graph $G$ extending $K$ by two vertices $x$ and $y$ with $E(G)-E(K)=\{x y, x z, y z\} \cup\left\{x x^{\prime}: x^{\prime} \in X\right\} \cup\left\{y y^{\prime}: y^{\prime} \in Y\right\}$. Note that $G \backslash x z / x y \cong K_{n}$. But no $K_{n}$-minor of $G$ uses $\{x y, x z, y z\}$ because contracting any other edge than $x y$ in $G$ results in a graph with more than one parallel pair of edges.

The next result allows us to derive triangle-roundedness in the class of regular matroids from triangle-roundedness in the classes of graphic and cographic matroids.

Theorem 5. If a family $\mathscr{F}$ of internally 4 -connected matroids with no triads is triangle-rounded both in the class of graphic and cographic matroids, then $\mathscr{F}$ is triangle-rounded in the class of regular matroids not isomorphic to $R_{10}$.

As $R_{10}$ has no $M\left(K_{5}\right)$-minor and $M\left(K_{5}\right)$ is internally 4-connected with no triads and trivially triangle-rounded in the class of cographic matroids, it follows from Theorems 4 and 5 that:

Corollary 6. $M\left(K_{5}\right)$ is triangle-rounded in the class of regular matroids.

All proofs are in the next section.

\section{Proofs}

In this section we prove the theorems. For matroids $M$ and $N$ we write $N<M$ to say that $M$ has a proper minor isomorphic to $N$. The notation $N \leq M$ means that $N<M$ or $N=M$. Next we state some results used in the proofs.

Lemma 7. (Whittle, [18, Lemma 3.6]) Let $M$ be a 3-connected matroid with elements $x$ and $p$ such that $\operatorname{si}(M / x)$ and $\operatorname{si}(M / x, p)$ are 3-connected, but si $(M / p)$ is not 3-connected. Then, $r(M) \geq 4$ and there is a rank-3 cocircuit $C^{*}$ of $M$ containing $x$ such that $p \in \operatorname{cl}_{M}\left(C^{*}\right)-C^{*}$.

Lemma 8. (Whittle [18, Lemma 3.7]) Let $C^{*}$ be a rank-3 cocircuit of a 3-connected matroid $M$ such that $p \in \operatorname{cl}_{M}\left(C^{*}\right)-C^{*}$.

(a) If $z_{1}, z_{2} \in C^{*}$, then $\operatorname{si}\left(M / p, z_{1}\right) \cong \operatorname{si}\left(M / p, z_{2}\right)$.

(b) If $N$ is a matroid and for some $x \in C^{*}, \operatorname{si}(N / x, p)$ is 3-connected with an $N$-minor, then $\operatorname{si}(N / z, p)$ is 3-connected with an $N$-minor for each $z \in C^{*}$.

Lemma 9. (Whittle [18, Lemma 3.8]) Let $C^{*}$ be a rank-3 cocircuit of a 3-connected matroid $M$. If $x \in C^{*}$ has the property that $c l_{M}\left(C^{*}\right)-x$ contains a triangle of $M / x$, then $\operatorname{si}(M / x)$ is 3-connected.

From Lemma 9, we may conclude:

Corollary 10. Let $M$ be a 3-connectced matroid with a triangle $T$ and a triad $T^{*}$ such that $T^{*}-T=\{x\}$ and $T-T^{*}=\{y\}$. Then $\operatorname{si}(M / x)$ and $\operatorname{co}(M \backslash y)$ are 3-connectced.

Lemma 11. ( $W u$, [19, Lemma 3.15]) If $I^{*}$ is a coindependent set in a matroid $M$ and $M \backslash I^{*}$ is vertically3-connected, then so is $M$. 
Using Seymour's Splitter Theorem (as stated in [11, Corollary 12.2.1]) and proceeding by induction on $i$ using Lemma11, we may conclude:

Corollary 12. Let $N<M$ be 3-connected matroids such that $M$ has no larger wheel or whirlminor than $N$ in case $N$ is a wheel or whirl respectively. Then, there is a chain of 3-connected matroids $N \cong M_{n}<\cdots<M_{1}<M_{0}=M$ such that for each $i=1, \ldots, n$ there is $x_{i} \in E\left(M_{i}\right)$ satisfying $M_{i}=M_{i-1} / x_{i}$ or $M_{i}=M_{i-1} \backslash x_{i}$. Moreover, for $I:=\left\{x_{i}: M_{i-1}=M_{i} / x_{i}\right\}$ and $I^{*}:=\left\{x_{i}: M_{i-1}=\right.$ $\left.M_{i} / x_{i}\right\}$

(a) I is an independent set and $I^{*}$ is a coindependent set of $M$.

(b) for each $1 \leq i \leq n, M /\left(I \cap\left\{x_{1}, \ldots, x_{i}\right\}\right)$ and $\left(M \backslash\left(I^{*} \cap\left\{x_{1}, \ldots, x_{i}\right\}\right)\right)^{*}$ are vertically3-connected.

Theorem 13. (Whittle, [18, Corollary 3.3]) Let $N$ be a 3-connected minor of the 3-connected matroid $M$. If $r(M) \geq r(N)+3$, then for each element $x$ such that $\operatorname{si}(M / x)$ is 3-connected with an $N$-minor, there exists $y \in E(M)$ such that $\operatorname{si}(M / y)$ and $\operatorname{si}(M / x, y)$ are 3-connected with $N$ minors.

Theorem 14. (Whittle [18, Lemma 3.4 and Theorem 3.1] and Costalonga [3, Theorem 1.3]) Let $k \in\{1,2,3\}$ and let $M$ be a 3-connected matroid with a 3-connected minor $N$ such that $r(M)-r(N) \geq k$. Then $M$ has a independent $k$-set $J$ such that $\operatorname{si}(M / x)$ is 3-connected with an $N$-minor for all $x \in J$.

Lemma 15. (Costalonga [4, Corollary 4]) Suppose that $N<M$ are 3-connected matroids with $r^{*}(M)-r^{*}(N) \geq 4$ and $N$ is cosimple. Then:

(a) $M$ has a coindependent set $S$ of size 4 such that $\operatorname{co}(M \backslash e)$ is 3-connected with an $N$-minor for all $e \in S$; or

(b) $M$ has distinct elements $a_{1}, a_{2}, b_{1}, b_{2}, b_{3}$ such that, $T_{s}:=\left\{a_{s}, b_{t}, b_{3}\right\}$ is a triangle for $\{s, t\}=$ $\{1,2\}, T^{*}:=\left\{b_{1}, b_{2}, b_{3}\right\}$ is a triad of $M$ and $\operatorname{co}\left(M \backslash T^{*}\right)$ is 3 -connected with an $N$-minor.

Next, we prove Theorem 3 and, after, using Theorem 3 , we prove Theorem 2 , For a nonnegative integer $k$, a $k$-segment of a matroid is a $k$-subset of a line of this matroid.

Proof of Theorem 3; Suppose that the result does not hold. This is, for each $N$-minor $N^{\prime}$ of $M$, $E(M)-E\left(N^{\prime}\right) \nsubseteq T$ and items (a) and (b) of the theorem do not hold. It is already known that $U_{2,4}$ is triangle-rounded [2], so, we may assume that $|E(N)| \geq 5$. The proof will be based on a series of assertions. First, note that it follows from the minimality of $M$ that:

(I). If, for $x \in E(M), \operatorname{si}(M / x)$ is 3-connected with an $N$-minor, then $x \in \mathrm{cl}_{M}(T)$.

(II). If $T^{*}$ is a triad and $T$ is a triangle of $M$ such that $T^{*}-T=\{x\}$, then $M \backslash x$ has no $N$-minor.

Subproof: Suppose the contrary. Let $T^{*} \cap T=\{a, b\}$. As $N$ is simple and cosimple, $M \backslash x / a \backslash b$ has an $N$-minor. But $M \backslash x / a \backslash b \cong M \backslash a, b / x$ and, therefore, $M / x$ has an $N$-minor. By Corollary 10, $\operatorname{si}(M / x)$ is 3-connected. By[(I), $x \in \operatorname{cl}_{M}(T)$. As $x \notin T$, then $T^{*}$ meets a 4-segment of $M$. This implies that $M \cong U_{2,4}$, a contradiction.

(III). If, for $x \in E(M), \operatorname{co}(M \backslash x)$ is 3-connected with an $N$-minor, then $x \in T$. 
Subproof: Suppose the contrary. Then $T \nsubseteq E(\operatorname{co}(M \backslash x))$ and therefore, there is a triad $T^{*}$ meeting $x$ and $T$. This contradicts (II).

(IV). If $\operatorname{si}(M / x)$ and $\operatorname{si}(M / x, y)$ are 3 -connected with $N$-minors, then so is $\operatorname{si}(M / y)$ and $x, y \in T$.

Subproof: First we prove that $\operatorname{si}(M / y)$ is 3-connected. Suppose the contrary. By Lemma 7. there is a rank-3 cocircuit $C^{*}$ such that $x \in C^{*}$ and $y \in \operatorname{cl}\left(C^{*}\right)-C^{*}$. By $[\mathrm{I}), x \in \operatorname{cl}(T)$. By orthogonality [11, Proposition 2.1.11], $T \subseteq \operatorname{cl}\left(C^{*}\right)$. As $r\left(C^{*}\right)=3$, there is $z \in C^{*}-\operatorname{cl}(T)$ and $T$ is a triangle of $M / z$ contained in $\operatorname{cl}_{M / z}\left(C^{*}\right)$. By Lemma 9 , $\operatorname{si}(M / z)$ is 3-connected and, by Lemma 8, $M / z$ has an $N$-minor. This contradicts (I), So, $\operatorname{si}(M / y)$ is 3 -connected.

$\operatorname{By}(\mathrm{I}), x, y \in \operatorname{cl}(T)$. If, for some $\{a, b\}=\{x, y\}, a \notin T$, then, as $M / b$ has an $N$-minor and $a$ is in a parallel pair of $M / b$, it follows that $M \backslash a$ has an $N$-minor. Moreover, in this case, $T \cup a$ is a 4-segment of $M$ and $M \backslash a$ is 3-connected with an $N$-minor, contradicting the minimality of $M$. Thus, $x, y \in T$.

(V). $r(M)-r(N) \leq 2$ and $r^{*}(M)-r^{*}(N) \leq 3$.

Subproof: If $r(M)-r(N) \geq 3$, then, by Theorem 14, there is an independent set $J$ of size 3 such that $\operatorname{si}(M / x)$ is 3-connected with an $N$-minor for all $x \in J$. So, there is $x \in J-\operatorname{cl}_{M}(T)$, a contradiction to (I), Thus, $r(M)-r(N) \leq 2$.

If $r^{*}(M)-r^{*}(N) \geq 4$, then Lemma[15applies. If item (a) of that Lemma holds, then we have an element $x \in E(M)-T$ such that $\operatorname{co}(M \backslash x)$ is 3-connected with an $N$-minor, contradicting (III), So, consider the elements given by item (b) of Lemma15, Let $s \in\{1,2\}$. Note that $M \backslash T^{*}$ is isomorphic to a minor of $M \backslash a_{s}$. By Corollary $10, \operatorname{co}\left(M \backslash a_{s}\right)$ is 3-connected with an $N$-minor and, therefore, $a_{s} \in T$ by (III), So, $a_{1}, a_{2} \in T$. By orthogonality between $T$ and $T^{*}, b_{3} \notin T$. As $M \backslash b_{3}$ has an $N$-minor, then, by (III), $\operatorname{co}\left(M \backslash b_{3}\right)$ is not 3-connected. By Bixby's Lemma [11, Lemma 8.7.3], $\operatorname{si}\left(M / b_{3}\right)$ is 3-connected. As $M \backslash T^{*}$ has an $N$-minor, then, so has $M / b_{3}$ and, therefore, $\operatorname{si}\left(M / b_{3}\right)$. By (I), $b_{3} \in \operatorname{cl}_{M}(T)$. But $b_{3}$ is in a triad and, therefore, in no 4-segment of $M$. So, $b_{3} \in T$, a contradiction.

(VI). The theorem holds if there is a wheel or whirl $W$ such that $N<W \leq M$.

Subproof: Let $W \cong M\left(W_{n}\right)$ or $W^{n}$. Since $N$ is a minor of $W$, then $N$ is isomorphic to a wheel or whirl. As $|E(N)| \geq 5$, then $|E(N)| \geq 6$ and $n \geq 4$. As $r(M)-r(N) \leq 2$ and $r(W)-r(N) \geq 1$, hence $r(M)-r(W) \leq 1$.

Suppose for a contradiction that $r(M)=r(W)$. So, for some coindependet set $J^{*}$ of $M$, $W=M \backslash J^{*}$. If $y$ is a non-spoke of $W$, then $W / y$ is vertically 3-connected by Corollary 10 , Moreover, $\operatorname{si}(W / y)$ has an $N$-minor and so does $\operatorname{si}(M / y)$. So, by Lemma 11, $\operatorname{si}(M / y)$ is 3connected with an $N$-minor for each non-spoke $y$ of $W$. By (I), all non-spokes of $W$ are in $\mathrm{cl}_{M}(T)$, contradicting the fact that they are the elements of a set with rank at least 3 in $W$.

Thus, $r(M)=r(W)+1$. Now, by Theorem 14, there is an element $x$ such that $\operatorname{si}(M / x)$ is 3-connected with an $W$-minor $W^{\prime}$. As $r(M / x)=r\left(W^{\prime}\right)$, there is coindependent set $I^{*}$ of $M / x$ such that $W^{\prime}=M / x \backslash I^{*}$. If $y$ is a non-spoke of $W^{\prime}, W^{\prime} / y=M / x, y \backslash I^{*}$ is vertically 3-connected and, by Lemma 11, so is $M / x, y$. By (IV), all non-spokes of $W^{\prime}$ are in $T$, a contradiction again. $\diamond$ 
As the theorem fails for $M, N$ and $T$, by (VI), there is no wheel or whirl $W$ such that $N<$ $W \leq M$ and the hypotheses of Seymour's Splitter Theorem hold for $M$ and $N$.

(VII). If $x \in E(M)$ and $\operatorname{si}(M / x)$ is 3-connected with an $N$-minor, then $x \in T$.

Subproof: Suppose the contrary. By[(I), $x \in \operatorname{cl}_{M}(T)$, which is a line with more than 3 points. As $M \backslash z$ is 3-connected for all $z \in \mathrm{cl}_{M}(T)-T$, then $M \backslash z$ has no $N$-minor if $z \in \operatorname{cl}_{M}(T)-T$. As $M / x$ has an $N$-minor, $\operatorname{cl}_{M}(T)=T \cup x$ and $M \backslash x$ has no $N$-minor. This implies that $r^{*}(M)-r^{*}(N) \geq 2$ as $T$ is in a parallel class of $M / x$.

Let us check that for each $z \in E(M)-x, \operatorname{si}(M / z)$ is not 3-connected with an $N$-minor. Suppose the contrary, by (I), $z \in \operatorname{cl}_{M}(T)$. So, $x$ is in the non-trivial parallel class $\operatorname{cl}_{M}(T)$ of $M / z$. Since $N$ is simple and $M / z$ has an $N$-minor, then $M / z \backslash x$, and, therefore, $M \backslash x$ have an $N$ minor. This is a contradiction to what we proved before. So, $x$ is the unique element of $M$ such that $\operatorname{si}(M / x)$ is 3-connected with an $N$-minor. By Theorem $14, r(M)-r(N)=1$.

Consider the structures defined as in Corollary 12, By what we proved, for all choices of $M_{1}, \ldots, M_{n}$, we have $I=\{x\}$ and $n=3$ or 4 . As $M / x$ has a parallel class with 3 elements, then $x=x_{3}$ or $x=x_{4}$, so, we have two cases to consider:

Case 1. We may pick $M_{1}, \ldots, M_{n}$ with $x=x_{n}$ : For all $y \in E(M)-x$ such that $M \backslash y$ is 3connected with an $N$-minor, we have $y \in T$ by (III). In particular this holds for each $y \in I^{*} \cup$ $\operatorname{cl}_{M}(T)-x$. So, $I^{*} \cup\left(\operatorname{cl}_{M}(T)-x\right) \subseteq T$. This implies the validity of the theorem and, in particular, of item (a). Contradicting the assumption that the Theorem fails for $M, N$ and $T$.

Case 2. Otherwise: Now, necessarily, $n=4$ and $x=x_{3}$. If $M^{\prime}:=M \backslash x_{1}, x_{2}, x_{4}$ is 3-connected, then, as $M^{\prime} / x$ is 3-connected, we could choose $x_{4}=x$, which does not hold in Case 2. Note, that $x$ is in a cocircuit with size at most two in $M^{\prime}$. As $M_{2}=M \backslash x_{1}, x_{2}$ is 3-connected, then $x$ is in a serial pair of $M^{\prime}$ with an element $z$. This implies that $M \backslash x_{1}, x_{2}, x_{4} / z \cong N$. By Lemma 11, $\mathrm{si}(M / z)$ is 3-connected with an $N$-minor. This contradicts the uniqueness of $x$ established before.

(VIII). If $\operatorname{co}(M \backslash x)$ and $\operatorname{co}(M \backslash x, y)$ are 3 -connected with $N$-minors, then $\operatorname{co}(M \backslash y)$ is 3 -connected and $x, y \in T$.

Subproof: Suppose the contrary. By (III) $\operatorname{co}(M \backslash y)$ is not 3-connected. By the dual of Lemma 7, there is a corank-3 circuit $C$ containing $x$ with $y \in \mathrm{cl}^{*}(C)-C$.

First assume that $C \neq T$. If $M$ has a 4 -cocircuit $D^{*}$ contained in $C \cup y$, then, as $\left|D^{*} \cap C\right| \geq 3$ and $T \nsubseteq \subseteq$, there is $z \in\left(D^{*} \cap C\right)-T$. So, $D^{*}-z$ is a triad of $M \backslash z$ contained in $\mathrm{cl}_{M \backslash z}^{*}(C-z)$ and, by the dual of Lemmas 8 and $9, \operatorname{co}(M \backslash z)$ is 3-connected with an $N$-minor. But this contradicts (III), Thus, $C \cup y$ contains no 4-cocircuit of $M$. But $r^{*}(C \cup y)=3$ and $y \in \mathrm{cl}^{*}(C)-C$, so $C \cup y$ is the disjoint union of a singleton set $\{e\}$ and a non-trivial coline $L^{*}$ containing $y$. By the dual of Lemmas 8 and 9 again, $\operatorname{co}(M \backslash e)$ is 3 -connected with an $N$-minor. By (III), $e \in T$. By the dual of Lemma 8, for some $f \in L^{*}, \operatorname{co}(M \backslash x, y) \cong \operatorname{co}(M \backslash f, y) \cong \operatorname{co}\left(M \backslash L^{*}\right)$ has an $N$-minor. Thus, $M / y$ has an $N$-minor and, by Bixby's Lemma, $\operatorname{si}(M / y)$ is 3-connected with an $N$-minor. By (VII), $y \in T$. Since $T$ meets $L^{*}$, it follows that $L^{*}$ is a triad and, as a consequence, $|C|=3$. By orthogonality, there is $g \in\left(L^{*} \cap T\right)-y \subseteq C$. Since $e \in(C \cap T)-L^{*}$, then $C \cup T$ is a 4-segment of $M$ meeting a triad, a contradiction since $M$ is 3-connected and not isomorphic to $U_{2,4}$. Therefore, $C=T$. 
Let $C^{*}$ be a cocircuit such that $y \in C^{*} \subseteq T \cup y$. If $C^{*}$ is a triad, we have a contradiction to (II) since $y \in C^{*}-T$ and $M \backslash y$ has an $N$-minor. So, $C^{*}$ is a 4 -cocircuit and $C^{*}=T \cup y$. By Bixby's Lemma, si $(M / y)$ is 3-connected. Since $y \notin T$, then $M / y$ has no $N$-minor by (VII), If $r(M)=r(N)$, then $N \cong M \backslash x, y$ and $M \backslash y$ is 3-connected, therefore $r(M)-r(N) \in\{1,2\}$.

For all 2-subsets $A$ of $T, M \backslash A$ has no $N$-minor because, otherwise, $y$ would be in the serial pair $C^{*}-A$ of $M \backslash A$ and $M / y$ would have an $N$-minor.

As $\operatorname{co}(M \backslash x, y)$ has an $N$-minor, hence $r^{*}(M)-r^{*}(N) \geq 2$. If $r^{*}(M)-r^{*}(N) \geq 3$, then, by Theorem 13, there is $z \in E(M)$ such that $\operatorname{co}(M \backslash z)$ and $\operatorname{co}(M \backslash x, z)$ are 3-connected with $N$ minors. By (III), $z \in T$. So, for $A:=\{x, z\} \subseteq T, M \backslash A$ has an $N$-minor, a contradiction. Therefore, $r^{*}(M)-r^{*}(N)=2$. To prove the theorem and item (b), we have to find an $N$-minor $N^{\prime}$ of $M$ with $E(M)-E\left(N^{\prime}\right) \subseteq T \cup y$. Consider a chain of matroids, sets and elements as in Corollary 12. Let $a$ and $b$, in this order, be the elements deleted from $M$ in order to get $M_{n}$ from $M$ as in the chain (recall that $r^{*}(M)-r^{*}(N)=2$ ). By Lemma11, $\operatorname{co}(M \backslash a)$ is 3-connected with an $N$-minor, hence, by (III), $a \in T$. It follows from Lemma 11, (IV), (V) and (VII) that $I \subseteq T$. We just have to prove now that $b=y$. Suppose the contrary. If $b \in T$, then, for $A:=\{a, b\} \subseteq T, M \backslash A$ has an $N$-minor, a contradiction, as we saw before. Thus, $b \notin T$ and, by (III), $\operatorname{co}(M \backslash b)$ is not 3-connected. So, $a$ and $b$ play similar roles as $x$ and $y$ and applying the same steps for $a$ and $b$ as we did for $x$ and $y$, we conclude that $D^{*}:=T \cup b$ is a cocircuit of $M$. By circuit elimination on $C^{*}:=T \cup y, D^{*}$ and any element $e$ of $T$, it follows from the cosimplicity of $M$ and from the orthogonality with $T$ that $(T-e) \cup\{y, b\}$ is a cocircuit of $M$. Therefore, $y$ is in a series class of $M \backslash a, b$, which has an $N$-minor. But this implies that $M / y$ has an $N$-minor, a contradiction. So, $b=y$ and (b) holds.

Now, consider the structures as given by Corollary 12. It follows from (IV), (V) and (VII) that $I \subseteq T$. If $r^{*}(M)-r^{*}(N) \leq 2$, it follows from (III) and (VIII) that $I^{*} \subseteq T$. This implies that $T \subseteq E(M)-E\left(N^{\prime}\right)$ for $N^{\prime}=M_{n}$ and the theorem holds in this case. So, we may assume that $r^{*}(M)-r^{*}(N)=3$. If $|I|=0$, then $N^{\prime}=M \backslash I^{*}$ and $M \backslash e$ is 3-connected with an $N$-minor for all $e \in I^{*}$ and by (III), $I^{*} \subseteq T$. So, $|I| \geq 1$. By Lemma[11 and (VIII), the elements of $I^{*}$ with the two least indices in $x_{1}, \ldots, x_{n}$ are in $T$. So, $|T \cap I| \leq 1$ and as $I \subseteq T,|I|=1$. Therefore, $n=4$. Since $I \subseteq T, x_{1} \notin I$ by the simplicity of $M_{1}$. If $I=\left\{x_{4}\right\}$, then $M \backslash e$ is 3-connected for all $e \in I^{*}$, a contradiction, as before. Therefore, $I=\left\{x_{3}\right\}$ or $\left\{x_{2}\right\}$. This implies that $T=\left\{x_{1}, x_{2}, x_{3}\right\}$.

By Theorem 14, there is a coindependent 3-set $J^{*}$ of $M$ such that $\operatorname{co}(M \backslash e)$ is 3-connected with an $N$-minor for all $e \in J^{*}$. By (III), $J^{*}=T=\left\{x_{1}, x_{2}, x_{3}\right\}$. If $T$ meets a triad $T^{*}$, then, for $f \in T^{*}-T$ and $e \in T \cap T^{*}$ we have that $M \backslash e$ and, therefore, $M \backslash e / f$, have an $N$-minor. But in this case, by Lemma 9 , si $(M / f)$ is 3 -connected with an $N$-minor, a contradiction to (IV), Thus, $T$ meets no triads of $M$. Next, we check:

(IX). We may not pick $M_{1}, M_{2}, M_{3}, M_{4}$ in such a way that $I=\left\{x_{3}\right\}$.

Subproof: Suppose the contrary. Then, we may pick the chain of matroids in such a way that $M_{4}=M \backslash x_{1}, x_{2} / x_{3} \backslash x_{4}$ with $I=\left\{x_{3}\right\}$. As $x_{4} \notin T$, by (III), $\operatorname{co}\left(M \backslash x_{4}\right)$ is not 3-connected and $M^{*}$ has a vertical 3-separation $\left(A, x_{4}, B\right)$. This is, both $A$ and $B$ are 3-separating sets of $M, x_{4} \in$ $\mathrm{cl}^{*}(A) \cap \mathrm{cl}^{*}(B)$ and $r^{*}(A), r^{*}(B) \geq 3$. So, $(A, B)$ is a 2-separation of $M \backslash x_{4}$, but $M \backslash x_{4}, x_{1}, x_{2} / x_{3}$ is 3 -connected and we may assume, therefore, that $\left|A-\left\{x_{1}, x_{2}, x_{3}\right\}\right|=|A-T| \leq 1$. As $|A| \geq 3$, then 
$|A \cap T| \geq 2$ and $A$ spans $T$. This implies that $Y=A \cup T$ is a 3-separating set of $M$. Moreover, $Y=T$ or $Y=T \cup y$ for some $y \in E(M)-T$.

Next we prove that $T \cup x_{4}$ is a cocircuit of $M$. Note that $x_{4} \in \operatorname{cl}^{*}(Y)$. If $Y=T$, then, as $T$ meets no triads, it follows that $T \cup x_{4}$ is a 4-cocircuit. So, we may assume that $Y=T \cup y$ for some $y \in E(M)-T$. If $r_{M}(Y)=2, Y$ is a 4-segment of $M$. As $M / x_{3}$ has an $N$-minor, then so has $M \backslash y$. In this case, $M \backslash y$ is 3-connected, contradicting(III) since $y \notin T$. Thus, $r_{M}(Y)=3$. Since $|Y|=4$ and $Y$ is 3-separating, it follows that $r^{*}(Y)=3=r^{*}(T)$. Now, $T$ cospans $y$, and as $T \cup y$ cospans $x_{4}$, it follows that $T$ cospans $x_{4}$ and $T \cup x_{4}$ is a cocircuit of $M$ since $T$ meets no triads.

As $T \cup x_{4}$ is a cocircuit of $M$, hence $T-\left\{x_{3}, x_{4}\right\}$ is a serial pair of $M_{2}=M \backslash x_{1}, x_{2}$ which is 3 -connected with at least 4 elements, a contradiction. Thus, (IX) holds.

Now, By (IX), $I=\left\{x_{2}\right\}$ for all choices of chains. This implies that there are no pair of elements $\{a, b\} \subseteq E(M)$ such that $M \backslash a$ and $M \backslash a, b$ are 3-connected with an $N$-minor. In particular, $M \backslash x_{1}, x_{3}$ is not 3-connected. But $M_{3}=M \backslash x_{1}, x_{3} / x_{2}$ is 3-connected. As $M \backslash x_{1}$ is 3-connected, then $x_{2}$ is in a serial pair $\left\{x_{2}, z\right\}$ of $M \backslash x_{1}, x_{3}$. Hence, $M / z$ has an $N$-minor. Since $M \backslash x_{1}$ is 3 -connected, it follows that $\left\{z, x_{2}, x_{3}\right\}$ is a triad of $M \backslash x_{1}$. But $T$ meets no triads of $M$ and, therefore, $C^{*}:=\left\{z, x_{1}, x_{2}, x_{3}\right\}=T \cup z$ is a 4-cocircuit of $M$. If $z \in \operatorname{cl}(T)$, then $r^{*}\left(C^{*}\right)=2$ and $C^{*}$ is a 2-separating set of $M$. This implies that $r(M)=r\left(C^{*}\right)=2$, contradicting the fact that $r(M)=r(N)+1 \geq 3$. So, $z \notin \operatorname{cl}(T)$ and $T$ is a triangle of $M / z$ contained in $C^{*}$. By Lemma 9 , $\operatorname{si}(M / z)$ is 3 -connected. But $M / z$ has an $N$-minor. By(VII), $z \in T$, a contradiction. This proves the theorem.

Proof of Theorem 2; If $|E(N)| \geq 4$, then, since binary matroids cannot contains 4-point lines nor circuits meeting cocircuits in an odd number of elements, then items (a) and (b) of Theorem 3 does not hold. In this case, Theorem 3 implies the theorem. So we may assume that $|E(N)| \leq 3$. If $N \supsetneqq U_{1,3}$, then, since $N$ is 3-connected, if follows that $N$ is isomorphic to a minor of $M^{\prime}:=M \mid T \cong U_{2,3}$. So, we may assume that $N \cong U_{1,3}$. In this case $M$ has a circuit $C$ meeting $T$ but different from $T$. Now, for any $e \in C-T$, the theorem holds for $M^{\prime}=(M \mid T \cup C) /(C-(T \cup e))$.

We define $K_{3,3}^{1,1}$ as the graph in Figure 1, The following lemma is a well-known result and is a straightforward consequence of Seymour's Splitter Theorem.

Lemma 16. If $G$ is a 3 -connected graph with a $K_{5}$-minor then, either $G \cong K_{5}$ or $G$ has a $K_{3,3}^{1,1}$ minor.

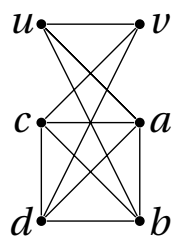

FIGURE $1 . K \cong K_{3,3}^{1,1}$ 
Proof of Theorem 4: We have to prove that for each 3-connected simple graph $G$ with a $K_{5}$ minor and for each triangle $T$ of $G, G$ has a $K_{5}$-minor using $E(T)$. Consider a counter-example $G$ with $|E(G)|$ as small as possible. By Theorem 2, we may assume that $E(G)-E\left(K_{5}\right) \subseteq E(T)$. As no edges may be added to $K_{5}$ in order to get a 3-connected simple graph, then $|V(G)|=6$ or 7 .

First suppose that $|V(G)|=7$. Now, $G$ is obtained from $K_{5}$ by two vertex splittings by edges $e_{1}$ and $e_{2}$ and, possibly, by the addition of an edge $e_{3}$ with $e_{1}, e_{2} \in E(T)$ and $e_{3} \in E(T)$ in case it is added. So, $e_{1}$ and $e_{2}$ are adjacent in $G$. When obtaining $G$ in this process, we split a vertex by $e_{1}$, then we split an endvertex of $e_{1}$ by $e_{2}$. If $x, y$, and $z$ are the three vertices incident to $e_{1}$ or $e_{2}$ in after these two splittings, then the sum of their degrees is 8 . So, in order to get $G$ with all vertices with degree at least 3 , the addition of $e_{3}$ is necessary. Note that this process describes that $G$ is obtained from $K_{5}$ by expanding a vertex into the triangle $T$ with vertices $x$, $y$ and $z$. Observe that there are two vertices $u, v \in V(T)$ with degree 3 . It is clear that for the edges $e, f \notin E(T)$ incident to $u$ and $v$ respectively, we have $\operatorname{si}(G / e, f) \cong K_{5}$.

So, we may assume that $|V(G)|=6$. By Lemma 16, up to labels, $G$ is obtained from $K \cong K_{3,3}^{1,1}$ (the graph in Figure11), by adding the edges of $E(T)-E(K)$. Since $K / u v \cong K_{5}$, then $u v \in T$ and we may assume without losing generality that $V(T)=\{u, v, a\}$, so $G=K+v a$. Now, it is clear that $G \backslash b a / u b$ is a $K_{5}$-minor of $G$ using $T$. This proves the theorem.

The following lemma has a slightly stronger conclusion than [11, Proposition 9.3.5] (beyond the conclusions of [11, Proposition 9.3.5], it states beyond that $R$ has a $K$-minor and describes the way it is obtained), but the proof for [11, Proposition 9.3.5] also holds for the following lemma.

Lemma 17. Let $R=K \oplus_{3} L$ be a 3-sum of binary matroids, where $K$ and $L$ are 3 -connected and $E(K) \cap E(L)=S$. Then there are $X, Y \subseteq E(L)-S$ such that $K \cong R / X \backslash Y$, where $K$ is obtained from $R / X \backslash Y$ by relabeling the elements $s_{1}, s_{2}$ and $s_{3}$ of $S$ in $K$ by respective elements $l_{1}, l_{2}$ and $l_{3}$ of $L$.

Proof of Theorem [5: Suppose that $R$ is a matroid contradicting the theorem minimizing $|E(R)|$. So, $R$ has a triangle $T$ and an $M$-minor for some $M \in \mathscr{F}$ but $M$ has no $\mathscr{F}$-minor using $T$. If $R$ is graphic or cographic, the theorem holds for $R$, so assume the contrary. By Seymour's Decomposition Theorem for Regular Matroids [11, Theorem 13.1.1], there are matroids $K$ and $L$ with at least 7 elements each, intersecting in a common triangle $S$ such that $R=K \oplus_{3} L$ with $L$ being 3-connected and $K$ being 3-connected up to parallel classes of size two meeting $S$. Under these circunstances, we may assume that $|E(K) \cap E(M)| \geq|E(L) \cap E(M)|$.

If $C$ is a cycle of $R$ meeting both $E(K)$ and $E(L)$, then there is $s \in S$ such that $(C \cap E(N)) \cup s$ is a cycle of $N$ for $N=K, L$. As we picked $L$ with no parallel pairs, it follows that $\operatorname{cl}_{R}(E(K)-S) \cap$ $E(L)=\varnothing$.

Let us first check that $K$ has an $M$-minor. Let $M=R / I \backslash I^{*}$ for some independent set $I$ and coindependent set $I^{*}$ of $R$. Since $\lambda_{M}(E(K) \cap E(M)) \leq \lambda_{R}(E(K) \cap E(M))=2$, then as $M$ is internally 4-connected, it follows that $|E(M) \cap E(L)| \leq 3$, and, moreover, $E(M) \cap E(L)$ is not a triad of $M$ because $M$ has no triads. This implies that $E(M) \cap E(L) \subseteq \mathrm{cl}_{M}(E(M)-E(L))$. By the format of the family of circuits of $R$, it follows that $E(M) \cap E(L) \subseteq \operatorname{cl}_{R}(E(K)-S)$, which is empty. So, $E(M) \subseteq E(K)$. By Lemma 17, there is a minor $K^{\prime}$ of $R$ obtained by relabeling the elements $s_{1}, s_{2}$ and $s_{3}$ of $S$ in $K$ by respective elements $l_{1}, l_{2}$ and $l_{3}$ of $L$. Consider the matroid $K^{\prime \prime}$ 
obtained from $K^{\prime}$ by contracting each $l_{i}$ for those indices $i \in\{1,2,3\}$ such that $s_{i} \in \operatorname{cl}_{L}(I \cap E(L))$. Now $K^{\prime \prime}$ is obtained from $R /(E(L) \cap I) \backslash\left(E(L) \cap I^{*}\right)$ by relabeling the remaining elements of $S$. This implies that $K^{\prime \prime}$ and, therefore, $K$ have $M$-minors.

If $T \subseteq E(K)$, then $K$ has an $\mathscr{F}$-minor using $T$ by the minimality of $R$. But $R$ has an $K$-minor using $T$ by Lemma 17 and this implies the theorem. So, $T$ meets $E(L)$. As $\operatorname{cl}_{R}(E(K)-S) \cap$ $E(L)=\varnothing$, it follows that $X:=T \cap E(L)$ has at least two elements. As $L$ is 3-connected and $\lambda_{L}(S)=\lambda_{L}(X)=2$, then $\kappa_{L}(S, X)=2$. By Tutte's Linking Theorem [11, Theorem 8.5.2], there is a minor $N$ of $L$ with $E(N)=S \cup X$ such that $\lambda_{N}(S)=2$. Hence:

$$
2=\lambda_{N}(S)=r_{N}(S)+r_{N}(X)-r(N) \leq 4-r(N) .
$$

So, $r(N) \leq 2$. But $r(N) \geq r_{N}(S) \geq \lambda_{N}(S)=2$. Also $r_{N}(X) \geq \lambda_{N}(X)=2$. This implies that $S$ spans $N$ and $X$ contains no parallel pairs of $N$. Now, each element of $X$ is in parallel with an element of $S$ in $N$. Therefore, for $N=L / A \backslash B$, we have that $R / A \backslash B$ is obtained from $K$ by relabeling the elements of $S$ by elements of $T$. So, $R / A \backslash B$ is 3-connected with $T$ as triangle and has an $M$-minor. By the minimality of $R, R / A \backslash B$ has an $\mathscr{F}$-minor using $T$ and this proves the lemma.

\section{SHARPNESS}

In this section we establish that Theorem 3 is sharp in the sense that all described cases may occur indeed.

First we construct an example for Theorem 3 with $E(M) \subseteq E(N) \cup T$. Consider a complete graph $K$ on $n \geq 14$ vertices. Let $X:=\left\{v_{i, j}: i=1,2,3\right.$ and $\left.j=1,2,3,4\right\}$ be a 12-subset of $V(K)$. Consider a triangle $T$ on vertices $u_{1}, u_{2}$ and $u_{3}$, disjoint from $K$. Let $G=K \cup T+\left\{u_{i} v_{i, j}: i=\right.$ $1,2,3$ and $j=1,2,3,4\}$. Define, for disjoint subsets $A$ and $B$ of $E(T), H:=G \backslash A / B, M:=M(G)$ and $N:=M(H)$ provided $H$ is a simple graph. For each $e \in E(G)-E(T), G / e$ has at least 3 parallel pairs; so, $|E(\operatorname{si}(M / e))| \leq|E(G)|-4<|E(H)|$ and, therefore $M / e$ has no $N$-minor. For $e$ incident to $v \in V(K)$, in order to get a minor of $G \backslash e$ with $|V(K)|-12$ vertices with degree $|V(K)|-1 \geq 13$ and 12 vertices with degree $|V(K)| \geq 14$ as in $H$, it is necessary to contract some edge out of $T$, thus $G \backslash e$ has no $H$-minor either. Note that we may pick $A$ with any size from 0 to 3 , We always may pick $B=\varnothing$, and provided $|A| \geq 1$ we may pick $B$ with size from 1 to $3-|A|$.

Now, let us construct an example satisfying item (a) of Theorem 3 , Let us pick $M$ as a restriction of the affine space $\mathbb{R}^{3}$. Consider a 4-subset $L:=\{a, b, c, x\}$ of an line $R$. Let $T:=L-x$. Now consider for each $y \in T$ a line $R_{y}$ meeting $L$ in $y$ in such a way that no three lines among $R, R_{a}, R_{b}$ and $R_{c}$ lay in a same plane. Let $m \geq 6$. For each $y \in T$, pick a $m$-subset $L_{y}$ of $R_{y}$ containing $y$. Let $M$ be the restriction of the affine space to $L \cup L_{a} \cup L_{b} \cup L_{c}$. Let $N=M / x \backslash a, b$ or $N=M / x \backslash T$. Note that it is not possible to get a rank-3 minor of $M$ with 3 disjoint $(m-1)$ segments by contracting an element other than $x$. So all $N$-minors of $M$ are minors of $M / x$ and therefore, deleting at least two elements of $T$ is necessary to get an $N$-minor since $T$ is a parallel class of $M / x$. So, this is the unique way to get an $M / x \backslash a, b$-minor of $M$. Moreover, deleting the element in the intersection of the three $m$-lines is the unique way to get and $M / x \backslash T$ from an $M / x \backslash a, b$-minor of $M$. 
Next, we construct an example satisfying item (b) of Theorem 3 , We denote by $M+e$ the matroid obtained by adding $e$ freely to $M$. Start with a projective geometry $P$ with $r(P) \geq$ 6. Let $F$ be a flat of $P$ with $4 \leq r(F) \leq r(P)-2$. Consider a copy $U$ of $U_{2,4}$ on ground set $T \cup x:=\left\{x, x_{1}, x_{2}, x_{3}\right\}$ with $(T \cup x) \cap E(P)=\varnothing$. Let $y$ be an element out of $E(P) \cup T \cup x$. Let $M$ be the matroid obtained by adding $y$ freely to the flat $F \cup T$ of $(P+x) \oplus_{2} U$. Note that $E(P)$ is a hyperplane of $M$ and, therefore $T \cup y$ is a 4-cocircuit of $M$. Define $N_{1}=M \backslash y / x_{2} \backslash x_{3}$ and $N_{2}:=N_{1} / x_{1}$. Note that $N_{1}=P+x_{1}$ and $N_{2}$ is the truncation of $P$ with rank $r(P)-1$ (the matroid on $E(P)$ whose independent sets are those independent sets of $P$ with size at most $r(P)-1)$.

Let $i \in\{1,2\}$ and $N=M / X \backslash Y$ be an $N_{i}$-minor of $M$. Note that $r^{*}(M)-r^{*}(N)=2$ and $r(M)-r(N) \in\{1,2\}$. For each $p \in E(P),|E(s i(M / p))|<\left|E\left(N_{i}\right)\right|$. Thus, no element $p \in P$ may be contracted in $M$ in order to get an $N_{i}$-minor. So, $X \subseteq T \cup y$.

Let us check that $T \cup y$ meets no circuit of $M$ with less than six elements other than $T$. Indeed, $M \backslash y$ is a two sum of a 4-point line on $T \cup x$ and a matroid with rank greater than five with $x$ as free element. Thus all circuits of $M \backslash y$ meeting $T$, except for $T$ itself, have more than five elements. Moreover, $M$ is obtained from $M \backslash y$ adding $y$ as a free element to a flat with rank greater than 4 and, therefore, all circuits of $M$ containing $y$ also have more than five elements. Hence, the triangles of $M / X$ are precisely the triangles of $P$. Moreover, those must be the same triangles of $N$ since all triangles of $N$ are triangles of $M / X$ and they occur in the same number. As deleting an element of $P$ from $M / X$ would result in a matroid with less triangles than $N_{i}$, it follows that $Y \subseteq T \cup y$. Hence, $E(P) \subseteq E(N)$ for each minor $N$ of $M$ isomorphic to $N_{1}$ or $N_{2}$.

Let us check that $M / y$ has no $N_{2}$-minor and, therefore, no $N_{1}$-minor too. Suppose for a contradiction that $N$ is an $N_{2}$-minor of $M / y$. We may assume that $N=M / y, x_{1} \backslash x_{2}, x_{3}$. Note that $x_{1}$ is a free element of the rank- $r_{P}(F)$ flat $F \cup x_{1}$ of $M / y \backslash x_{2}, x_{3}$. This implies that $N \mid F$ is a truncation of rank $r_{P}(F)-1$ of the rank- $r_{P}(F)$ projective geometry $F$. But, as $N_{2}$ is the rank$(r(P)-1)$ truncation of $P$ and $r_{P}(F) \leq r(P)-2$, then all rank- $\left(r_{P}(F)-1\right)$ flats of $N_{1}$ are projective geometries and so is $F$, a contradiction.

Now, for $i=1,2$, each $N_{i}$-minor of $M$ is in the form $M \backslash y, x_{i} / A$ with $1 \leq i \leq 3$ and $A$ being an $i$-subset of $T-x_{i}$. Let $A$ be a 2-subset of $T$. As we proved for $A=\left\{x_{2}, x_{3}\right\}$, it follows that $M \backslash A$ has no $N_{2}$-minor. Moreover, for $x_{k} \in T-A$ it is clear that $y$ is not a free element of $M \backslash A / x_{k}$, which, therefore, is not isomorphic to $N_{1}$. Thus, $M \backslash A$ has no minor isomorphic to $N_{1}$. This implies that $M \backslash A$ has no minor isomorphic to $N_{2}$ neither.

\section{ACKNOWLEDGEMENTS}

The authors thank the Visiting Scholar Fund of the Department of Mathematics and Statistics at Wright State University for the support.

\section{REFERENCES}

[1] R. E. Bixby, $\ell$-matrices and a characterization of binary matroids, Discr. Math. 8 (1974), 139-145.

[2] T. Asano, T. Nishizeki and P. D. Seymour, A note on non-graphic matroids, J. Combin. Theory Ser. B 37 (1984) 290-293.

[3] J. P. Costalonga, On3-connected minors of3-connected matroids and graphs, European J. Combin. 33 (2012), 72-81. 
[4] J. P. Costalonga, A splitter theorem on 3-connected matroids, European J. Combin. 69 (2018) 7-18.

[5] C. R. Coullard, Counterexamples to conjectures on 4-connected matroids, Combinatorica, 6 (1986), 315-320.

[6] J. Kahn, A problem of P. Seymour on nonbinary matroids, Combinatorica, 5 (1985), 319-323.

[7] T. Moss, A minor-based characterization of matroid 3-connectivity, Adv. Appl. Math 50 (2013) 132-141.

[8] J. G. Oxley, On the intersections of circuits and cocircuits in matroids. Combinatorica 4 (1984) 187-195.

[9] J. G. Oxley, On nonbinary 3-connected matroids. Trans. Amer. Math. Soc. 300 (1987) 663-679.

[10] J. G. Oxley, A characterization of certain excluded-minor classes of matroids. European J. Combin. 10 (1989) 275-279.

[11] J. G. Oxley, Matroid theory, Second Edition, Oxford graduate texts in mathematics, Oxford University Press, 2006.

[12] J. G. Oxley and T. J. Reid, The smallest rounded sets of binary matroids, European J. Combin. 11 (1990) 47-56.

[13] T. J. Reid, Triangles in 3-connected matroids, Discrete Mathematics 90 (1991) 281-296.

[14] P. D. Seymour, A note on the production of matroid minors. J. Combin. Theory Ser. B 22 (1977) $289-295$.

[15] P. D. Seymour, Decomposition of regular matroids, J. Combin. Theory Ser. B 28 (1980), 305-359.

[16] P. D. Seymour, On minors of non-binary matroids, Combinatorica 1 (1981), 387-394.

[17] P. D. Seymour, Minors of 3-connected matroids, European J. Combin. 6 (1985), 375-382.

[18] G. Whittle, Stabilizers of classes of representable matroids, J. Combin. Theory Ser. B 77 (1999), 39-72.

[19] H. Wu, On contractible and vertically contractible elements in 3-connected matroids and graphs, Discrete Math. 179 (1998), 185-203. 